\title{
Prediction of tissue thermal damage
}

\author{
Xin $\mathrm{Li}^{\mathrm{a}, *}$, Yongmin Zhong ${ }^{\mathrm{a}}$, Aleksandar Subic ${ }^{\mathrm{b}}$, Reza Jazar ${ }^{\mathrm{a}}$, Julian Smith ${ }^{\mathrm{c}}$ and Chengfan $\mathrm{Gu}^{\mathrm{a}}$ \\ ${ }^{a}$ School of Engineering, RMIT University, Bundoora, VIC, Australia \\ ${ }^{\mathrm{b}}$ Swinburne Research and Development, Swinburne University of Technology, Hawthorn, VIC, \\ Australia \\ ${ }^{\mathrm{c}}$ Monash Medical Centre, Monash University, Clayton, VIC, Australia
}

\begin{abstract}
This paper presents a method to characterize tissue thermal damage by taking into account the thermal-mechanical effect of soft tissues for thermal ablation. This method integrates the bio-heating conduction and non-rigid motion dynamics to describe thermal-mechanical behaviors of soft tissues and further extends the traditional tissue damage model to characterize thermal-mechanical damage of soft tissues. Simulations and comparison analysis demonstrate that the proposed method can effectively predict tissue thermal damage and it also provides reliable guidelines for control of the thermal ablation procedure.
\end{abstract}

Keywords: Tissue thermal damage, bio-heat transfer, tissue thermo-mechanical behaviors, thermal ablation

\section{Introduction}

As an effective alternative of traditional tissue cancer treatments, such as surgical resection and chemotherapy, thermal ablation technique uses energy sources including radiofrequency, laser, and microwaves to treat cancerous tissues. It has the advantages of minimally invasive, shortened recovery time, and controlled side effect to surrounding healthy tissues. The success of this therapy requires the accurate characterization of tissue damage under thermal load. Currently, most of the existing studies only rely on thermal-induced temperature elevation to predict tissue damage area, ignoring the effect of thermal-mechanical deformation during this procedure [1-4]. However, even a relatively small variation in thermal-induced mechanical deformation can lead to various effects such as altered production of hormones, suppressed immune response, and protein denaturation [5]. In fact, such thermo-mechanical effect can lower the barrier (energy level) to unfolding protein from a relative native state to a transition state [6], leading to further damage to biological tissues. Therefore, it is an absolute necessity to take into account the tissue damage due to the thermal-mechanical effect to accurately characterize tissue damage for thermal ablation.

Until now, the research on thermal-mechanical behaviors of biological tissues has received great attention. Shen has developed a thermo-mechanical deformation model using quasi-steady state thermoelasticity to study skin burn [7]. Xu treated skin tissue as a laminated composite structure, and used a sequentially coupled thermo-mechanical model to discuss the thermo-mechanical characteristic of skin tissue under thermal load [8]. In general, most of the existing studies are dominated by theoretical analysis to identify thermal-induced mechanical properties of soft tissues, while there has been very limited

\footnotetext{
${ }^{*}$ Corresponding author: Xin Li, School of Engineering, RMIT University, Bundoora, VIC 3083, Australia. E-mail: jiner286@ gmail.com.
}

0928-7329/16/\$35.00 @ 2016 - IOS Press and the authors. All rights reserved

This article is published online with Open Access and distributed under the terms of the Creative Commons Attribution NonCommercial License. 
research on real-time computer modeling of tissue thermal-mechanical behaviors for thermal ablation. Recently, the authors' research group has reported such a method for modeling thermal-mechanical deformations of soft tissues by combining bio-heat transfer and continuum mechanics for thermal ablation [9]. But, the issue of tissue thermal-mechanical damage was not studied yet. The Arrhenius burn integration is commonly used to describe tissue thermal damage $[1,10,11]$. However, this model is based on a chemical rate process, and the thermal-induced mechanical effect due to thermal variation is ignored.

This paper presents a new method to predict tissue thermal damage by taking into account the thermalmechanical effect of soft tissues. It establishes a thermal-mechanical model by combing bio-heat transfer theories, constitutive elastic material law under thermal load and non-rigid motion dynamics to characterize the thermal-induced mechanical behaviors of soft tissues. Based on this, a tissue damage model is established by taking into account the thermo-mechanical effect of soft tissues. This model adopts thermal-induced strain energy in the traditional tissue damage model to represent the thermal-mechanical damage of soft tissues under thermal load. Simulation results and comparison analysis have been conducted to evaluate the performance of the proposed method.

\section{Proposed method}

\subsection{Thermal-mechanical model}

The bio-heat diffusion within a tissue body without thermo-elastic coupling, is given as,

$$
\rho C \frac{\partial T}{\partial t}=k\left(\frac{\partial^{2} T}{\partial x^{2}}+\frac{\partial^{2} T}{\partial y^{2}}+\frac{\partial^{2} T}{\partial z^{2}}\right)+C_{b} \omega_{b} \rho_{b}\left(T_{b}-T\right)+Q_{m}+Q_{e x t}
$$

where $\rho$ is the tissue density, $C$ the tissue specific heat, $k$ the tissue thermal conductivity, $T$ the temperature at time $t, C_{b}$ the specific heat of circulated blood, $\omega_{b}$ the blood perfusion, $\rho_{b}$ the blood density, $T_{b}$ the arterial temperature, $Q_{m}$ defines the generated metabolic heat, $Q_{e x t}$ the heat source. In this article, the heat source is only set at those points where heat energy is applied.

The governing equation for the non-rigid mechanics of motion can be written as

$$
\rho \ddot{u}_{i}=\sigma_{i j, j}+F_{i}
$$

From constitutive elastic material law under thermal loads, the stress tensor is related to the strain tensor, or the displacement components, and temperature change $\theta$ as,

$$
\sigma_{i j}=\mu\left(u_{i, j}+u_{j, i}\right)+\lambda u_{k, k} \delta_{i j}-\beta \theta \delta_{i j}
$$

where $\delta_{i j}$ is the Kronecker's symbol, which equals 1 for $i=j$, and 0 for $i \neq j$.

Equation (2) can be further written as,

$$
\rho \ddot{u}_{i}=\left(\mu\left(u_{i, j}+u_{j, i}\right)+\lambda u_{k, k} \delta_{i j}-\beta \theta \delta_{i j}\right)_{, j}+F_{i}
$$

where $\beta=\alpha(3 \lambda+2 \mu), \lambda$ and $\mu$ are the Lame constants, and $F_{i}$ is the exerted external force.

To solve the above partial differential equations, it is necessary to determine the boundary conditions under consideration. Here, (a) Thermal, the Dirichlet boundary conditions is chosen to determine the character of heat interchange over the boundary. i.e. the specified temperature on the boundary $\Omega$ is set to be $310 \mathrm{~K}$. (b) Elastic, fixed conditions is set at the tissue model surface, so that all boundaries cannot move freely. For an initial conditions at $t=0 \mathrm{~s}$, we assume $T=310 \mathrm{~K}$, and no strain inside the tissue model before applying heat. 


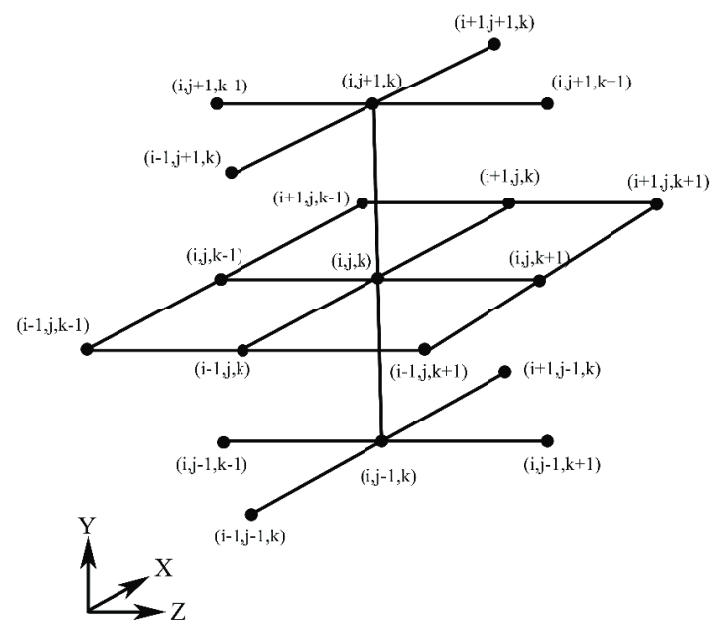

(a)

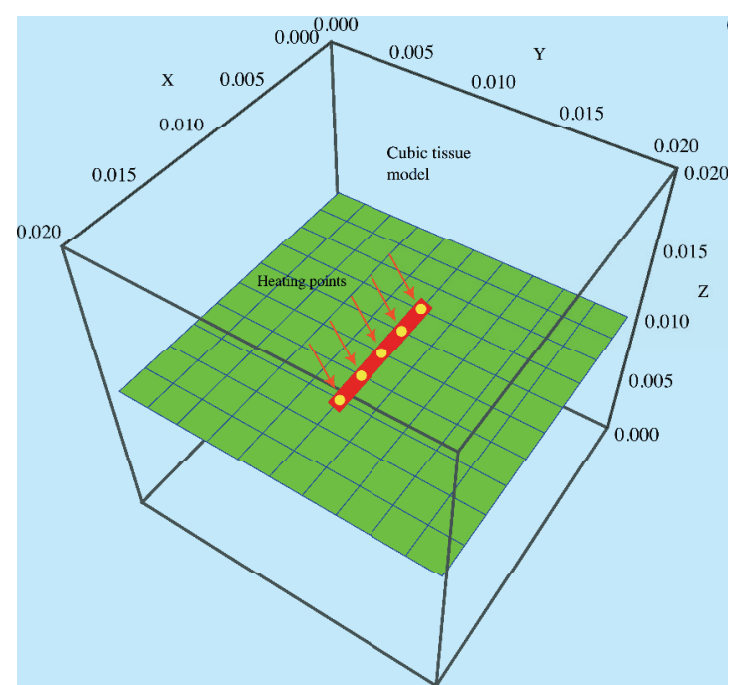

(b)

Fig. 1. (a) A cubic grid; (b) Dimensions of the cubic tissue model and positioning of heating sources.

\subsection{Thermal-mechanical damage model}

Soft tissues endure thermal stress and deformation due to temperature variation. As proteins in tissues can be denatured at relatively small activation energy, biological tissues are more easily damaged under thermal-induced stress and deformation. This means that the thermal-induced strain energy helps proteins to overcome the barrier from a relative native state to a transition state during any denaturant. Based on this observation, this paper incorporates the thermal-induced strain energy in the traditional tissue damage model of Arrhenius integration to characterize tissue thermal damage. The proposed thermalmechanical tissue damage model is described as

$$
\Omega=\zeta \int_{0}^{t} \exp \left(-\frac{\Delta E-E_{\text {mechanical }}}{R T_{(x, t)}}\right) d t
$$

where $\zeta$ is a material parameter (frequency factor or damage rate factor), $\Delta E$ is the activation energy, $R$ is the universal gas constant, and $t$ is the exposure time at a given temperature $T_{(x, t)}$. $E_{\text {mechanical }}$ is the unit strain energy, which is represented by Eq. (6), where $V_{\text {mol }}$ stands for the molar volume of target tissue.

$$
E_{\text {mechanical }}=\frac{1}{2} V_{\text {mol }} \sum_{i, j=1}^{3} \sigma_{i j} \varepsilon_{i j}
$$

\section{Results and discussions}

Simulations were conducted to investigate the performance of proposed model in terms of a cubic tissue model in the size of $20 \mathrm{~mm} \times 20 \mathrm{~mm} \times 20 \mathrm{~mm}$, as shown in Fig. 1(b). The tissue model contains 


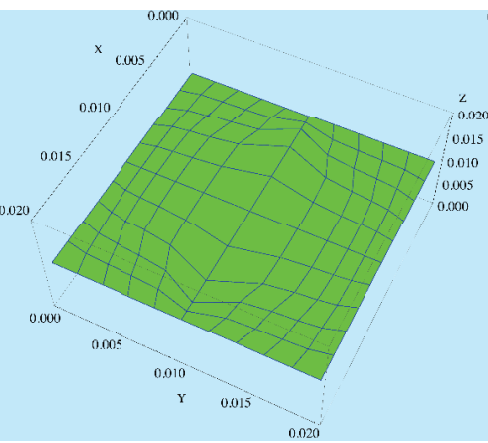

(a)

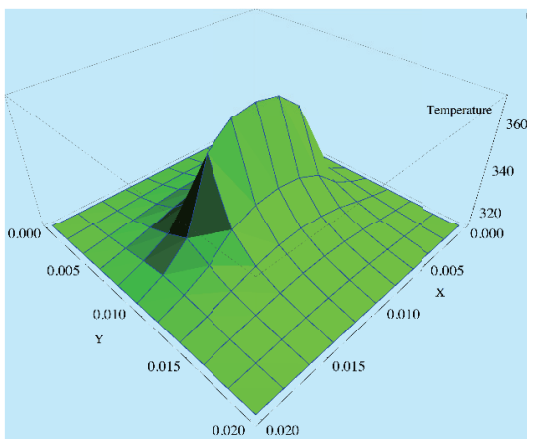

(b)

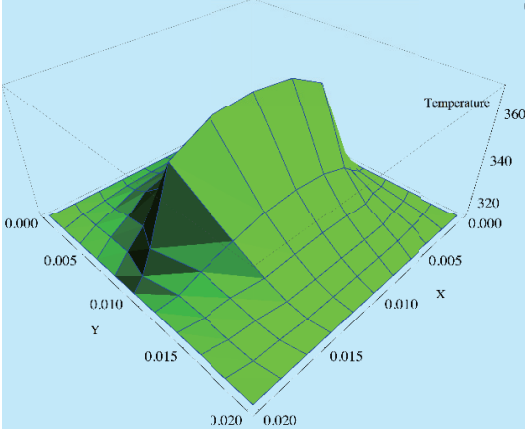

(c)

Fig. 2. (a) Displacement at plane $Z=0.01 \mathrm{~m}$; (b) Temperature rise at plane $Z=0.01 \mathrm{~m}$ for the model without considering tissue deformation; (c) Temperature rise at plane $Z=0.01 \mathrm{~m}$ for the model considering tissue deformation.

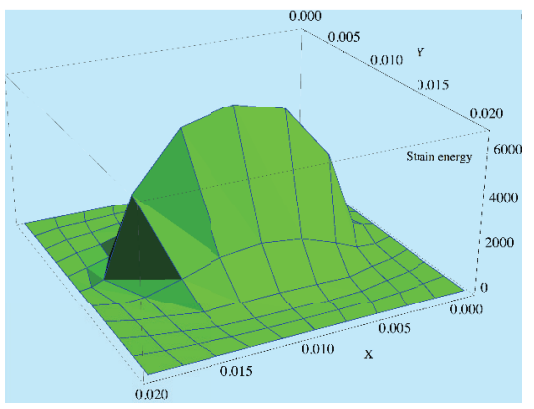

(a)

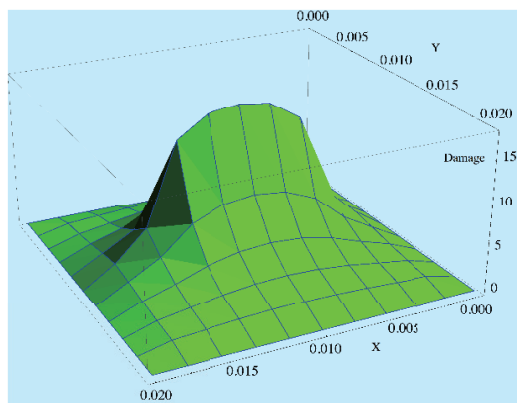

(b)

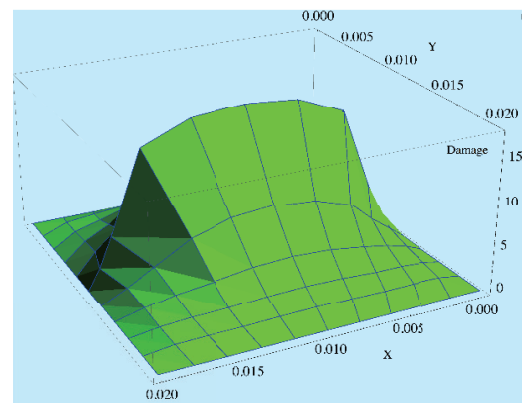

(c)

Fig. 3. (a) Strain energy at plane $Z=0.01 \mathrm{~m}$; (b) Damage at plane $Z=0.01 \mathrm{~m}$ for the model without considering tissue deformation. (c) Damage at plane $Z=0.01 \mathrm{~m}$ for the model considering tissue deformation. Note: in Figs 3(b) and (c), the plotted tissue damage is scaled to $\Omega^{\prime}=\log \Omega+4$.

1331 nodes and 1000 cubic elements (see Fig. 1(a)). The needle used in the thermal ablation treatment is modeled as a straight line with five evenly spaced points at the front head for generating heat energy. As indicated in Fig. 1(b), the needle is injected into a position where the middle of five heating points is located at the center of plane $Z=0.01 \mathrm{~m}$. The needle generates thermal energy through the five points at $2.0 \times 10^{7} \mathrm{~W} / \mathrm{m}^{3}$ for a time period of $500 \mathrm{~s}$ to heat up the tissue model, and there is no external force applied to the tumor model. The values of relevant thermal and mechanical parameters of the simulated tissue are the same as in our previous study (9). For the comparison analysis purpose, simulations were conducted under the same conditions for both models. One considers the thermal-mechanical effect of soft tissues and the other does not.

Figure 2(a) shows the displacement of plane $Z=0.01 \mathrm{~m}$ at the end simulation time of 500 seconds. Considering the position and distribution of applied heating sources, for the inner points located symmetrically with respect to the heating line, the deformations move outwards (along the $Y$ axis) consistently from high temperature to low temperature.

Comparing the temperature changes for both tissue models with and without considering the thermalinduced mechanical effect, Figs 2(b) and (c) show the temperature rise at the end of the exposure time $500 \mathrm{~s}$ at plane $Z=0.01 \mathrm{~m}$ where thermal energy is directly applied. It can be seen that for both models, the maximum elevation of temperature is around $367.362 \mathrm{~K}$ and happens at the center of the heating line. However, due to the thermal-induced mechanical effect, the temperature field decreases relatively 
slowly along the heat propagation direction ( $Y$ axis). That is, the tissue deformation obviously changes the temperature distribution within the target tissue, while there still is only one peak temperature located at the center of the heating sources.

To study strain energy distribution within the tissue model as a result of temperature variation, analysis has been further conducted at plane $Z=0.01 \mathrm{~m}$. Figure 3(a) gives the strain energy distribution within this plane at the end of the heating period. It is observed that the point located at the center of the heating line encounters the maximum strain energy of $6788.257 \mathrm{~J} / \mathrm{m}^{3}$. Figures 3 (b) and (c) compare the predicted damage distribution of the two models with and without considering deformed tissue, at the same selected plane $Z=0.01 \mathrm{~m}$. It can be seen that for the model considering thermal induced tissue deformation, the tissue endures a higher level of damage, while the resultant damage field follows the temperature distribution, decreasing relatively slowly along the heat propagating direction ( $Y$ axis).

\section{Conclusions}

This paper presents a new method to characterize tissue thermal damage for thermal ablation. This method takes into account thermal-induced mechanical effect in tissue thermal damage. It establishes the models to describe tissue thermal-mechanical behaviors and further characterize tissue thermal damage. Simulations and comparison analysis for both models with and without considering thermal-mechanical deformation demonstrate that the proposed method can account for tissue thermal damage more accurately. Future research will focus on experimental verification to further evaluate the performance of the proposed method with respect to real tissues.

\section{References}

[1] Prakash P. Theoretical modeling for hepatic microwave ablation. Open Biomed Eng J. 2010; 4: 27-38.

[2] Watanabe H, Yamazaki N, Kobayashi Y, Miyashita T, Ohdaira T, Hashizume M, et al. Estimation of intraoperative blood flow during liver rf ablation using a finite element method-based biomechanical simulation. 2011 Annual International Conference of the Ieee Engineering in Medicine and Biology Society (Embc). 2011: 7441-7445.

[3] Wu T, Li P, Shao Q, Hong J, Yang L, Wu S. A simulation-experiment method to characterize the heat transfer in ex-vivo porcine hepatic tissue with a realistic microwave ablation system. Numerical Heat Transfer, Part A: Applications. 2013; 64(9): 729-43.

[4] Rattanadecho P, Keangin P. Numerical study of heat transfer and blood flow in two-layered porous liver tissue during microwave ablation process using single and double slot antenna. International Journal of Heat and Mass Transfer. 2013; 58(1): 457-70.

[5] LAU CP, TAI YT, Lee PW. The effects of radiofrequency ablation versus medical therapy on the quality-of-life and exercise capacity in patients with accessory pathway-mediated supraventricular tachycardia: a treatment comparison study. Pacing and Clinical Electrophysiology. 1995; 18(3): 424-32.

[6] Rounsevell R, Forman JR, Clarke J. Atomic force microscopy: mechanical unfolding of proteins. Methods. 2004; 34(1): $100-11$.

[7] Shen WS, Zhang J. Modeling and numerical simulation of bioheat transfer and biomechanics in soft tissue. Mathematical and Computer Modelling. 2005; 41(11-12): 1251-65.

[8] Xu F, Lu T. Introduction to skin biothermomechanics and thermal pain, Springer; 2011.

[9] Li X, Zhong Y, Jazar R, Subic A. Thermal-mechanical deformation modelling of soft tissues for thermal ablation. BioMedical Materials And Engineering. 2014; 24(6): 2299-310.

[10] Henriques F, Jr, Moritz A. Studies of thermal injury: I. The conduction of heat to and through skin and the temperatures attained therein. A theoretical and an experimental investigation. The American Journal of Pathology. 1947; 23(4): 530.

[11] Pearce JA. Comparative analysis of mathematical models of cell death and thermal damage processes. International Journal of Hyperthermia. 2013; 29(4): 262-80. 